\title{
La obra literaria en la enseñanza de las lenguas y los géneros textuales
}

\author{
Dora Riestra*
}

\begin{abstract}
Resumen
Este artículo trata una cuestión teórico-práctica que atañe a los contenidos lingüísticos y las obras literarias en la enseñanza. La diversidad de disciplinas de las Ciencias del lenguaje y el consiguiente efecto de fragmentación del objeto de enseñanza, pone a la Didáctica de la lengua y la literatura frente a la tarea de reunir en la formación docente los contenidos de disciplinas de referencia y áreas de conocimiento diferentes. Además, el texto literario, como obra de arte, tiene aspectos culturales y técnicos que nos plantea otra integración de contenidos, necesaria en la conformación del objeto de enseñanza. Frente a estos desafíos de articulación teórica de la relación entre el lenguaje, las lenguas y las artes del texto, nos basamos en los programas de Saussure, Vygotski y Voloshinov, que orientan la búsqueda epistemológica en la problemática didáctica. Desde esta perspectiva abordamos los conceptos de signo y de género como principios organizadores de reformulación del objeto de enseñanza. Las formulaciones de lnteraccionismo socio-discursivo (BRONCKART, 1997) y de las Ciencias de la cultura (RASTIER, 2012) son los marcos teóricos que nos permiten delimitar la relación entre el texto/enunciado y la obra de arte literaria.

Palabras claves: didáctica, lenguaje y literatura, signo, obra literaria, género textual.
\end{abstract}

\section{The Literary Work in Teaching Tongues and Texts Genres}

\begin{abstract}
This paper reviews the linguistic contents taught and the place assigned to literary works when teaching language. The diversity of disciplines in Language Sciences has produced fragmentation of the object of teaching; as a result, the Didactics of Language and Literature should include contents of language and literature in teacher training courses. With regard to the literary text as a work of art, the issue is both cultural and technical in nature, two aspects which also pose the need for integration in the object of teaching. The fact that the programs of Saussure, Vygotski and Voloshinov are still valid regarding the challenges of the theoretical articulation of the relationship between language and tongues guides the methodological search for tackling the issue of didactics. The concepts of sign and genre are organizing principles of the didactic object to be reformulated. The approaches of Sociodiscursive Interactionism (Bronckart, 1997) and the Science of Culture (Rastier, 2012) enable us to define the relationship between the text/statement and the literary work of art.
\end{abstract}

Key words: didactics, language and literature, sign, literary work, text genres

\section{La historia de la literatura: la linealidad}

La investigación actual de la problemática de la Didáctica de la lengua y la literatura en Argentina nos presenta la disgregación y atomización de los objetos de enseñanza, tanto en la formación docente universitaria como en la escolaridad secundaria y primaria.

Consideramos que se trata de un problema originado, en parte, en los intentos aplicacionistas de los modelos teóricos lingüísticos y que, desde la década del 70, ha ido profundizándose en la separación de contenidos de lengua y literatura, formulados para la enseñanza de esta área de conocimiento escolar.

El problema didáctico que relevamos tiene dos aspectos epistemológicos: a) qué lengua enseñamos y b) qué lugar le otorgamos a la literatura en la enseñanza de la misma.

Además, por ser dos aspectos indisociables de entidades diferentes, nos encontramos frente a una tarea compleja de discriminación en la investigación didáctica.

Partimos de la concepción de lengua como técnica históricamente determinada (Coseriu, 1991) y código semiológico no no-creativo (De Mauro, 1986), concepciones lingüísticas de referencia saussureana ambas, así como de la concepción de literatura como arte a ser transmitida en la escuela, en la articulaciónentre ética y estética (Rastier, 2012; Vygotski, 2005).

El cruce disciplinar que proponemos apunta, necesariamente, al análisis de los posibles sesgosdel objeto de estudio y sus consecuencias didácticas,

*Endereço eletrônico: driestra@unrn.edu.ar 
puesto que los contenidos de enseñanza transpuestos serán determinantes de la práctica docente frente a los conceptos de lenguaje, texto, lengua, gramática, obra artística o, mejor dicho, se trata de resituar los abordajes del objeto de enseñanza en un orden epistemológico consciente de su efecto formativo, lo que implica rupturas disciplinares.

Por otra parte, la literatura como arte puede abordarse desde diversas perspectivas: enseñamos historia de la literatura, enseñamos a analizar obras literarias, enseñamos a clasificar géneros literarios o, nada de esto y algo diferente y superador.

El objeto de enseñanza a delimitar implica tomar decisiones respecto del texto, desde el género y de la obra, como dos procesos didácticos lineales en sus dimensiones filogenéticas y ontogenéticas. Concretamente: se plantea la disyuntiva de partir del género o partir de la obra como dos opciones con direcciones diferentes en la propuesta didáctica.

El análisis histórico de la enseñanza de la Literatura en Argentina (Setton, 2004; Vivante, 2006;López Casanova, 2008; Nieto, 2010) pone el foco en la crítica a la posmodernidad y sus efectos fragmentarios. No obstante la precisión sostenida en el análisis lineal (iniciado por Bombini, 2004),un cierto descriptivismo abstracto de estos trabajos cierra la búsqueda de posibles salidas en la transmisión cultural, cuyo análisis es una tarea teórico-práctica pendiente en el campo de las ciencias humanas-sociales, de la que no está exenta la Didáctica de las lenguas y las literaturas.

Conocer la obra literaria implica conocer la historia literaria, a la vez que conocer el texto sin estudiar la composición discursiva del mismo es un imposible o un absurdo como objetivo didáctico.

El planteo de Rastier (ob cit) acerca de la "obra que utiliza y construye el lenguaje" es coincidente con el concepto de enseñanza de literatura que propone Coseriu (1987), quien sostenía que "no pueden separarse la enseñanza del lenguaje y de la literatura porque el lenguaje y la literatura constituyen, en un sentido que veremos, una forma única de la cultura, aunque como dos polos diferentes de esta forma". En realidad, avistaba la complejidad teórico-práctica del problema didáctico al sostener que "la deseable unificación debe plantearse dos veces: para el lenguaje y la literatura y para la gramática", es decir desde el punto de vista de la cultura y el lenguaje, por una parte $\mathrm{y}$, por otradesde el lenguaje y la lengua, lo que llevado a campos disciplinares mencionaba como lingüística y ciencia literaria.

No obstante, en la tradición occidental, el papel atribuido a la Teoría Literaria y dentro de ésta a la Semiología y a la Teoría de la Comunicación, incluso a la Teoría de los textos nos sitúa frente a un panorama de análisis de préstamos disciplinares muy amplio, a la vez que enriquecedor, bastante complejo.

Otro aspecto didáctico a considerar frente a la enseñanza de la literatura es el trabajo con la percepción del objeto, con la emoción y la razón, las dos características del lenguajear humano, según Maturana (2003,2005).Transmitir emociones y racionalidades significa poner en juego la propia sensibilidad artística y la propia capacidad de reflexionar en el lenguaje con las lenguas; en definitiva, tenemos que poner sobre la mesa de trabajo las concepciones revisitadas de Saussure y Voloshinov (2010) respecto de los signos lingüísticos, que operan como signos ideológicos en los enunciados/textos, cuya elaboración discursiva tiene determinados formatos psicolingüísticos (los tipos de discurso) y, al mismo tiempo se organizan desde los géneros de la palabra (Voloshinov, 2009) o géneros textuales (Bronckart, 2007).

Dirá Rastier (2012, p. 14): "Todavía hoy la separación entre lingüística y literatura parece insuperable, como si la literatura no fuera un arte de lenguaje", por lo que propone un plan general para superar la separación en el estudio de los textos "literarios y científicos", separación de nuestra época, en la que fuimos cayendo sin percibirlo, como nos sucede en los campos disciplinares con las especificidades que se transforman en desviaciones epistemológicas.

Como profesores, debemos mostrar a los alumnos los discursos funcionando en los textos en tanto objetos de construcción de sentido, de construcción estilística de cada autor, es decir, aunar sensibilidad con racionalidad, estética con construcción "lenguajera", el hecho artísticoliterario y el hecho textual-discursivo.

Por lo tanto, la reflexión linguística en la enseñanza del texto literario como transmisión cultural es la posibilidad de incorporar los humanos jóvenes al mundo de los adultos, enseñando a captar mundos sociales (valores históricos que se reconstruyen). Es la escuela particularmente la que puede hacer conocer y descubrirlos en el carácter de obras artísticas, es decir, compartir mundos construidos discursivamente en su composición estética.

Para transmitir una obra literaria, necesariamente tenemos que conocerla como tal en 
sus aspectos históricos, filológicos, retóricos y lingüísticos. ¿Qué significa este conocer?

Se trata de reflexionar sobre las formas lingüísticas en función de la composición artística: en nuestro caso, partimos de los tipos de discurso (ap Bronckart) que operan en las obras de literatura como textos y conforman los estilos de las mismas. $\mathrm{Si}$ el primer problema es la relación lengualiteratura en la enseñanza, el segundo problema es de otra índole: se trata de la discusión acerca de la enseñanza de los géneros y de la obra literaria, como un problema de la planificación, discusión que puede darse simultáneamente en el centro de dos ámbitos disciplinares: el de la didáctica y el de la teoría literaria. La delimitación del objeto de enseñanza literatura como arte del lenguaje, a la vez que obra de la cultura, en formulación de Rastier, nos plantea la necesidad de definir algo previo: qué es la obra literaria para los profesores de Lengua y Literatura hoy.

Observamos, a partir de investigaciones didácticas que la obra literaria se lee superficialmente para responder cuestionarios acerca de las características o simplemente se lee por leer, sin guía del profesor ni explicaciones técnicas, a veces con impresiones subjetivas o comentarios de la crítica literaria. Las dimensiones del texto literario en su riqueza lingüística en función del efecto cultural producido no se han sintetizado en propuestas didácticas, aunque sí se presentan los géneros como regularidades de la composición en el carácter de componentes descriptivos de las obras (Riestra, 2011, a y b).

El diálogo disciplinar que propone Rastier (ob cit) entre la lingüística, la retórica y la hermenéutica y, por otra parte, la articulación de los campos literarios y lingüísticos, como estudios diversos que se necesitan, es un aporte para el abordaje didáctico de una obra literaria. La hermenéutica como interpretación unificadora nos permite una orientación en las miradas posibles y complementarias frente a la obra literaria, el contexto de producción (la época) y el de las recepciones posteriores (el nuestro frente a los clásicos). El enfoque filológico apuntaría a sostener la necesidad de la contextualización histórica de las obras que es, en definitiva, la referencia de la linealidad histórica, una necesidad metodológica para la enseñanza de la literatura, ya que sin el eje de la interpretación histórica, no hay posibilidad de enseñar y transmitir valor [lo que se abordará más adelante].El análisis lingüístico y retórico estaría encuadrado entre la interpretación y la contextualización histórica de la obra. En realidad, se trata de revisitar la estilística en sus posibilidades analíticas y de transposición didáctica.

\section{La totalidad y la fragmentación del sentido de la obra en los textos: la discrecionalidad}

Frente a la problemática de la elección de obras y enseñanza de géneros observamos que se plantean dos criterios diferentes, no articulados en la práctica:

a) por una parte la atención está puesta en las regularidades del género textual específico para analizar y entender las continuidades y rupturas, propias de los géneros como enunciados transmisores de signos ideológicos sociales (Voloshinov, ob cit);

b) por otra parte, la obra literaria elegida consiste en un recorte socio-histórico determinado ética y estéticamente, que tendrá las características singulares del estilo de la época y del autor, lo que significa poner la atención en un aspecto discrecional opuesto a la regularidad genérica y que, además, en la actualidad esuna tendencia retroalimentada por las descripciones de las tipologías textuales aplicadas a la enseñanza como método en los textos escolares.

Constituye todo un desafío de acciones de lenguaje poder abarcar las cuatro dimensiones del signo lingüístico en un recorrido semántico, pragmático, sintáctico y expresivo, como la pluriplanaridad lingüística que formula De Mauro (2005) para expresar, explicar y transmitir sentidos.

En el inicio del proceso didáctico está la obra y su contextualización imprescindible como objeto de enseñanza; lo genérico sería la herramienta lingüístico-comunicativa en la que la dimensión discursivo-textual es la clave que permite entender la obra en su composición para valorarla estética y éticamente como producto socio-histórico, hecho singular y colectivo a la vez.

La cuestión didáctica a resolver es, en consecuencia, una solución de compromiso, puesto que el género estaría en función de la obra y no a la inversa, como se ha planteado hasta ahora para organizar una secuencia didáctica (Bronckart y Schneuwly,1991; Schneuwly, 1994; Dolz; Noverraz e Schneuwly, 2004).

\section{La sincronía y la diacronía de la recepción de la literatura como problema hermenéutico: mutabilidad e inmutabilidad de la obra}


Sin dudas, podemos decir que el problema epistemológico de etiquetar una época responde a consideraciones de índoles estéticas y éticas.

En este sentido no tenemos respuestas que aclaren respecto de la necesidad de etiquetar las épocas con conceptos significativos para la formación de los niños y jóvenes. Los criterios cronológicos sabemos que han sido esquemáticos y arbitrarios en una dirección ideológica centralista que aún no fue reemplazada por otra concepción que nos permita articular la perspectiva sincrónica con la diacrónica.

En la formación de profesores las referencias de la teoría literaria, así como de la crítica literaria no parecen contribuir a la práctica profesional con criterios definidos en función de qué sentidos literarios poner en juego en un diseño cultural consciente de la enseñanza de la lengua y la literatura para la próxima generación (Del Río \& Álvarez, 2003). Es decir, el tecnicismo abstracto o la subjetividad extrema en la valoración de las obras permanecen como conocimientos ajenos al proceso de enseñanza-aprendizaje de los alumnos del profesorado (Riestra, 2012).

Debemos tener en cuenta que el lugar del lector ha cambiado entre la segunda mitad del siglo XX y esta porción del siglo XXI, como sostiene Umberto Eco en el prólogo a la obra de Omar Calabrese, La era neobarroca. Reconoce que hoy existe otro lector, en términos culturales, es decir, se refiere al contexto de recepción de la obra literaria en los últimos veinte años. El papel de la escuela para introducir la recepción del sentido del texto literario también ha cambiado, por lo que hace falta reconstruir culturalmente el diseño del espacio literario escolar, en particular, para salir de la trampa de la llamada "literatura infanto-juvenil".

Calabrese (1999, p.11) propone la búsqueda de "las huellas de la existencia de un gusto de nuestro tiempo por los objetos más dispares, desde la ciencia hasta las comunicaciones de masa, desde la literatura hasta la filosofía, desde el arte hasta los comportamientos cotidianos". El término neobarroco que acuña este autor para identificar nuestra época casi a nivel de percepción colectiva es, en realidad, una categoría para analizar formas de articulación de diversas manifestaciones culturales. Se pronuncia como crítico de lo antimetódico y propone la búsqueda de racionalidad en el análisis. Esto nos es muy útil, en un sentido didáctico, porque, si bien cuestiona los criterios de organización por épocas de la historia de la cultura, plantea el concepto de continuidad y ruptura como dos aspectos del análisis a considerar para construir el objeto teórico como "objeto de cultura comunicable". La propuesta de analizar objetos culturales de una época como fenómenos de comunicación (música, cine, literatura, televisión, arquitectura, pintura, etc.) dotados de forma y estructura subyacente es una búsqueda de caracteres comunes entre sí.

La estética y la ética son ejes a los que Calabrese (ob cit) hace referencia exacta, es decir, no se trata de buscar solamente formas, sino de encontrar los valores de época en esas formas, por eso menciona categorías estéticas y axiológicas para hacer el análisis.

Como principio organizador, la búsqueda de los valores de época constituye una posibilidad de aproximación al concepto de contexto de producción del texto, desde la perspectiva del interaccionismo sociodiscursivo.

Asimismo, el concepto de género de Voloshinov (obcit) nos aporta y se articula con este enfoque de continuidad y ruptura en las dimensiones diacrónica y sincrónica, ya que los géneros se organizan en determinadas situaciones y los enunciados que los componen contienen los signos ideológicos que se retroalimentan colectiva e individualmente.

A esta altura nos preguntamos acerca de los saberes referenciales de la crítica literaria que serían útiles para enseñar literatura. La respuesta que demos a la pregunta nos llevará por diversos caminos posibles: a) enseñar nociones de la crítica literaria a los jóvenes que tendrán de este modo herramientas teóricas para abordar las obras literarias; b) no enseñar nada técnico, sino transmitir síntesis interpretativas realizadas por críticos literarios, es decir, la divulgación de la crítica literaria para formar lectores actualizados; c) realizar modelizaciones de los géneros literarios y encontrar las características de los mismos en las obras elegidas; d) entrar en contacto con obras singulares de la historia de la literatura para interpretar la estética de una época y realizar nuevas interpretaciones estéticas a través del conocimiento lingüístico.

Estas cuatro opciones podrían ser el marco problemático posible para debatir qué enseñar hoy en las clases de literatura. No obstante, ninguna de esas opciones parece profundizar en la problemática de la transmisión de valor.

Para quienes nos planteamos la problemática de la transmisión de la literatura como obra y como textos-documentos se nos presenta un 
problema hermenéutico a formular y a resolver, en la medida en que debemos transmitir nuestra interpretación posible y la de algunos autores de referencia. ¿Es posible no tomar posición frente a una obra de arte? ¿La interpretación del profesor debe guiar la lectura de la obra? ¿Cómo abordar una obra producida en un contexto socio-histórico distante sin la interpretación previa del profesor o sin las claves interpretativas? ¿Desde dónde construir esas claves?

La necesidad de afinar los conceptos de género y obra literaria en función de la transmisión cultural escolar nos lleva, en la práctica del aula de literatura, a realizar el análisis de época de los géneros y su valor ético cultural en el contexto de producción. Es decir, no se trata hacer "clasificaciones" de géneros ni "descripciones" de obras con categorías de autor, funciones, etc. El objetivo es más complejo porque nos ocupa la simultaneidad de los aspectos a considerar en la enseñanza, como hemos venido formulando, desde los aportes de las diversas disciplinas y los autores mencionados.

Sí, podemos sostener que en esta revisión del objeto de enseñanza, el modelo de la arquitectura textual formulado por Bronckart (1997, 2008), cobra una nueva significación como referencia teórica para la propuesta didáctica de Uso-Sentido- Forma que hemos desarrollado.

\section{El estilo y la eficacia del lenguaje humano con la técnica de las lenguas: el significado y la arbitrariedad radical respecto del significante}

Enseñar a conocer y reconocer el estilo como efecto artístico de las palabras en la obra literaria es una posibilidad didáctica que nos ofrece la estilística revisitada, a partir de las nuevas lecturas de Saussure, las que no pudimos leer en el siglo XX, quizá por el mismo efecto de su revolución semiológica no comprendida desde el paradigma representacionalista del lenguaje. Un siglo después, la relación indisociable del significado y el significante se nos presenta en el carácter desdoblado de la semiosis, a la vez que la posible articulación de las relaciones gramaticales en el proceso particular de la textualización literaria.

Volver a leer a Charles Bally, resignificado como contemporáneo de Saussure, contribuye a redimensionar esta noción de estilo, descartada por el paradigma didáctico del "enfoque comunicativo". La contradicción que explicitara entre expresión y comunicación al formular que "el objeto de la estilística es la expresión hablada y no el hecho pensado" fue leída como todo Saussure, en términos de dicotomía. Para Bally(1967) se trataba de una característica del hablar y el pensar, como acciones del habla: la palabra, desde el punto de vista del sujeto hablante es medio de acción y expresión, desde el punto de vista del sujeto entendiente es fuente de impresiones y reacciones.

En la didáctica de las lenguas esta contradicción fue tomada en sentido literal, como dos "funciones del lenguaje" cuando, en realidad son dos aspectos de la actividad de lenguaje, concebida ésta con otra complejidad, profundizando en el sentido de la doble situación.

Para Bally (1941/1967) sentimiento e intelecto dividen al sujeto hablante. En consecuencia, los procedimientos lexicológicos son considerados en este marco como los medios de expresión directa y los procedimientos sintácticos como los medios más importantes de expresión indirecta. Esta posición recupera los conceptos saussureanos acerca de la doble esencia del signo para volver de otro modo al texto literario.

En términos de Bally (ob cit), un mismo signo tiene muchos valores y cada valor es expresado por muchos signos. Esta clave de la estilística nos permite abordar el texto literario como unidad de sentido y unidad de comunicación, en la perspectiva semiológica para recuperar, desde allí, el arte construido con las palabras, en nuestra propia lengua o en la traducción de otras lenguas.

Se trata de una revisión teórica con efectos en la práctica, ya que el arte se percibe en obras concretas (generalmente textos escritos, en nuestro caso), por lo que "didactizar" no consiste en transmitir valor artístico en abstracto, ni comentarios o generalizaciones sobre las obras.

La perspectiva de educación artística, que Vygotski (ob cit) formuló con claridad en los años 20 del siglo pasado, se nos perdió en las aulas entre los conceptos de la linguística y de la teoría literaria y creímos que clasificando géneros, tipos de textos o tipos de funciones, etc, podría aprenderse a leer y escribir mejor, a percibir el valor artístico y a vivenciarlo.

En síntesis, si enseñar literatura es enseñar a valorar el arte como actividad creadora y crítica de los humanos, estamos ante la situación novedosa, aunque antigua y muchas veces olvidada, de abordar con responsabilidad la formación artística con la literatura en la escuela. Los conceptos vygotskianos de educación estética y sublimación del arte contribuyen para situar la búsqueda del efecto de la 
propuesta didáctica. Pero, ¿en qué consiste hoy la formación estética? ¿Qué mostrar de la obra? ¿Qué sentido podría tener empezar por la forma más allá de ser una práctica puramente aplicacionista, alejada de la lectura literaria?

Son preguntas que pretenden desnaturalizar lo que se hace en las aulas, que es el modo como se reproduce el disgusto por la literatura, algo ajeno "que hay que aprender" por obligación curricular. Así se aborda genéricamente "la gauchesca", "el género policial", el llamado "género no ficcional", etc, todos términos vacíos que se reproducen hoy en las escuelas primarias y secundarias. Además, muchos de los géneros de textos que hoy circulan por las aulas de Lengua y Literatura no constituyen modelos sociales intencionalmente buscados para formar a nuestros alumnos, son el resultado de la "era neobarroca", el residuo cultural que parece mayoritariamente aceptado por docentes y alumnos, "lo posible". Les pregunto a mis alumnos $\mathrm{y}$ me pregunto si realmente queremos transmitir esos textos como obras literarias, les pregunto quién decide el criterio de selección estético y ético. No hay respuestas.

Si bien la noción de géneros textuales o géneros de la palabra, que se originan en las actividades humanas, según Voloshinov (ob cit), es una noción que, pese a haber sido erróneamente atribuida a Bajtin, revolucionó la teoría, la aplicación de ésta en las aulas se resuelve "clasificando" aspectos del género, es decir, se volvió una tipología textual simplificada.

El estudio del proceso de generación de los textos como la "ingeniería de los textos humanos en las actividades" no logró insertarse en la formación docente.

Como sostienen Dolz\&Gagnon (2008), apelando a los criterios de legitimidad, pertinencia y solidaridad, la modelización o el modelo didáctico designa las características de un género, aún provisorias en función de orientar las prácticas de enseñanza de los profesores. Pero, a pesar de reconocer la finalidad didáctica de la descripción de las características del género que debe presentar siempre un carácter operacional, esta propuesta no logró superar el aplicacionismo del modelo didáctico, que operó como modelo teórico a ser aplicado (Riestra, 2012)

$\mathrm{Si}$ partimos del principio teórico voloshinoviano, retomado por Bronckart (2007, 2008), la composición genérica de todo texto, ya sea oral o escrito, situado en una práctica comunicativa y social, es lo que permite ligar el contexto y la situación. Por lo tanto, el género como un principio organizador del texto, es una herramienta generada colectivamente para poder decir individualmente, con la mediación de una lengua funcional, como técnica semiótica histórica (Coseriu, 1986). Esta lengua funcionalimplica el conocimiento de una gramática dentro de un sistema de posibilidades lingüísticas y bajo una norma social. Todos estos objetos de enseñanza encajados y ordenados en niveles de conocimientos diversos constituyen los contenidos que debería transmitir la escolaridad y que en la práctica resultan inabarcables.

De allí lo fragmentario, quizá. No obstante, desde una perspectiva teórica saussureana, De Mauro plantea esta inabarcabilidad como la condición necesaria de la creación lingüística $o$, dicho en sus términos, se trata de trabajar en la indeterminación semántica de la lengua histórica concreta con las infinitas extensiones de significado.

Es en este sentido que adoptamos, desde el enfoque interaccionista socio-discursivo un trayecto didáctico centrado en el texto objeto empírico y objeto artístico para desde esa empiria cultural llegar a la noción más amplia de género.

La inversión epistemológica del enfoque teórico es una necesidad didáctica en este caso, por lo que consideramos necesario profundizar en esta discusión.

En nuestra concepción didáctica planteamos enseñar la obra contextualizada histórica y socialmente. Partimos de conocer el contexto de producción (USO), su efecto cultural en la época, las influencias en la formación del autor, su valoración por la crítica y, sobre todo el SENTIDO que la obra tiene hoy para nosotros. Desde la transmisión del sentido estético y ético que podamos atribuirle, solamente allí, cobra valor la obra literaria en su FORMA.

Asimismo, proponemos en este trayecto didáctico de USO-SENTIDO-FORMA, una simplificación delos tres enfoques de abordaje de los textos propuestos por Rastier (ob cit) que son el enfoque filológico, el enfoque retórico y el enfoque hermenéutico en una integración. Sin caer en posibles reduccionismos, creemos que la hermenéutica sin la perspectiva de la ética y la estética sería un procedimiento formal (la técnica de las "preguntas" al texto), ya que los textos artísticos nos interpelan en nuestras motivaciones profundas y esto no puede dejarse de lado; es decir, el efecto catártico del arte nos conduce aproducir un género ensayístico que debemos aprovechar también como 
actividad de exposición oral y de escritura a partir de una obra estudiada.

Como puede inferirse de este planteo, no sería posible enseñar literatura sin transmitir la obra literaria, como tampoco podría concretarse sin conocimiento profundo de la obra y sus contextos de producción y recepción (gusto de época). Asimismo, si no conocemos la gramática de la lengua funcional no podremos trasmitir el valor artístico del texto, no alcanzaría el intento de transmitir la propia emoción que produce la obra en el profesor; en la enseñanza de la literaturaes imprescindible articular la percepción y la explicación con la emoción y la razón para enseñar a valorar la obra de arte.

Además, como las cuestiones de estilo, siendo efectos de sentido, sólo pueden verse desde la forma, necesitamos de los aportes poéticos retóricos acumuladosy transmitidos (diacrónica y sincrónicamente) en cada lengua (qué nos quiere "decir", para qué, por qué y con qué recurso lo hizo el autor, etc), por lo que el pasaje de uso a forma y de sentido a forma es recurrente, se entra y se sale de la forma del texto para aprehender el sentido y el contexto del autor en conjunción con el de nosotros, lectores, sobre todo, al "explicar" el efecto de emoción con palabras de la literatura, aun cuando pase el tiempo y la recepción cambie, en parte, debido a las estéticas.

Desde el concepto de De Mauro (ídem) de indeterminación semántica de la lengua, la extensión del significado del signo lingüístico apela a la acepción autonímica o reflexiva por la que las palabras y signos se refieren a sí mismos, es decir a la reflexión lingüística o a la metalengua.

Transpuesto didácticamente esto correspondería a trabajar desde el sentido los significados (lexemas morfológicos) y las formas (oposiciones y asociaciones en la linealidad sintagmática).

En lo que respecta al concepto de continuidad y ruptura planteado por Calabrese (ob cit), como dos aspectos del análisis a tener en cuenta para construir el objeto teórico como "objeto de cultura comunicable", su utilidad didáctica conceptual es coincidente con el concepto de Voloshinov (ídem) respecto del género: ante la obra literaria nos preguntamos qué ruptura significa históricamente y con qué aspecto de la historia tiene una continuidad. En lo que concierne a los géneros textuales $\mathrm{y}$, particularmente, los literarios constituyen una posibilidad de transmitir las recuperaciones y reformulaciones culturales e ideológicas en la historia de una cultura y una nación. Este principio metodológico reemplazaría el enfoque historiográfico o el de las épocas arbitrariamente elaboradas en decisiones políticas dentro de paradigmas, hoy, cuestionados.

Retomamos la concepción del interaccionismo socio-discursivo para considerar la literatura como preconstructo socio histórico, estudiado en las dimensiones textuales y discursivas del lenguaje, donde los signos funcionan en los textos como útiles semióticos. Las unidades verbales "textos" son consideradas como unidades psicológicas y los segmentos discursivos (tipos de discurso) como unidades psicolingüísticas infraordenadas en los textos. A su vez, los significados que tienen los signos son momentáneamente estables, por lo que es a través de esos signos en los textos, en constante movimiento, que se construyen los mundos representados y por la transformación permanente de los mundos construidos y explicados verbalmente, los contextos van cambiando y vamos entendiendo de manera diferente en diferentes épocas.

\section{Algunas conclusiones}

Los dos aspectos el problema didáctico que relevamos, es decir, la concepción de la lengua como técnica histórica y el lugar de la literatura como arte a ser transmitida en la escuela constituyen dos aspectos indisociables y, al mismo tiempo dos entidades diferentes, que comenzamos a investigar didácticamente.

Puede verse en ambos casos que el objeto a delimitar implica tomar decisiones respecto del texto desde el género y como obra, como dos procesos lineales diversos en sus dimensiones filogenéticas y ontogenéticas.

En lo que respecta al texto literario, en el carácter de obra de arte, se nos presenta un problema de índole tanto cultural como técnico, por lo que estos dos aspectos nos llevan a reformular la integración de los contenidos de Lengua y Literatura en el objeto de enseñanza.

Los conceptos de continuidad y la ruptura ante la obra literaria como "objeto de cultura comunicable" y ante los géneros como formas lingüísticas en las que se inscriben los textosnos plantean la necesidad de buscar una solución metodológica de compromiso para enfocar la obra literaria en la escuela, superando el aplicacionismo tanto de modelos lingüísticos como de nociones de 
la teoría literaria en abstracto.

El estilo como objeto de enseñanza a partir de la perspectiva semiológica (un mismo signo tiene muchos valores y cada valor es expresado por muchos signos) es un desafíodidáctico para abordar el texto literario como unidad de sentido y unidad de comunicación y, desde la indeterminación semántica como condición de la lengua funcional, abrir la elaboración del sentido del texto y recuperar el arte construido con las palabras, en nuestra propia lengua o en la traducción de otras lenguas.

La obra como objeto de enseñanza está en el inicio del proceso de una secuencia didáctica; su contextualización imprescindible implica tanto el aporte de los enfoques filológicos, hermenéuticos y retóricos; lo genérico sería el principio organizador de los textos literarioscomo herramienta comunicativa que permite percibir y entender la obra en su composición discursivo-textual y valorarla en las dimensiones estética y ética, como producto socio-histórico, hecho singular y colectivo a la vez.

\section{Referências}

BALLY, Ch. El lenguaje y la vida. Buenos Aires:Losada, 1967.

BOMBINI, G. Los arrabales de la literatura. La historia de la enseñanza literaria en la escuela secundaria argentina (1860-1960). Buenos Aires: Miño y Dávila, 2004.

BRONCKART, J.-P. Conferencia inaugural: La actividad verbal, las lenguas $y$ la lengua;reflexiones teóricas y didácticas. Barcelona, 18 y 19 de enero 2007: I jornadas de Investigación sobre $\mathrm{La}$ educación lingüística y literaria en entornos plurilingües. 2007b.

BRONCKART, J-P. Desarrollo del lenguaje y didáctica de las lenguas. Buenos Aires: Miño y Dávila, 2007a.

BRONCKART, J.-P. y SCHENUWLY,B. "La didáctica de la lengua materna: el nacimiento de una utopía indispensable", Textos de Didáctica de la Lengua y de la Literatura, $N^{\circ}$, julio1996.

CALABRESE, O. "Introducción" y Capítulo 1: "El gusto y el método". La era neobarroca, 1999.
COSERIU, E., La innovación en la enseñanza de la lengua $y$ la literatura. Madrid:MEChttp://www.romling.unituebingen.de/c oseriu/publi/coseriu225.pdf, 1987.

COSERIU. E. El hombre y su lenguaje, Madrid: Gredos, 1986.

DE MAURO, T. Minisemántica. Madrid: Gredos, 1986.

DE MAURO, T. Guida all'uso delle parole. Roma: Editori Riuniti, 2003.

DE MAURO, T. Primera lección sobre el lenguaje. México: SigloXXI, 2005.

DEL RÍO, Á. Los futuros humanos desde el pasado: Genética Cultural y Diseño Cultural. Revista CEPAOS, N4, Vol II, 2003.

DOLZ, J.; NOVERRAZ, M.;SCHNEUWLY, B. Seqüências didáticas para o oral e a escrita:presentação de umprocedimento. In: ROXO, R.; CORDEIRO, G. Gêneros orais e escritos na escola. Campinas: Mercado de Letras, 2004, p. 95128.

DOLZ, J. \&GAGNON, R. Le genre du texte, un outil didactique pour développer le langage oral et écrit. Pratiques,137/138.2008, p. 179-198,

LÓPEZ CASANOVA, M. Introducción. Literatura argentina y pasado reciente. Buenos Aires: UNGS, 2008.

MATURANA, H. MATURANA R., H. "Lenguaje y realidad: el origen de lo humano", en: Desde la Biología a la Psicología. Buenos Aires: Lumen, 2003.

NIETO, F. Escuela media, canon y consumos culturales, Educación, lenguaje y sociedad. Bs. As.: UNGS, 2010.

NUÑEZ RUIZ. La enseñanza y el aprendizaje de la literatura hoy. La educación literaria. Madrid: Síntesis, 2001.

RASTIER, F. Artes y Ciencias del texto. Madrid: Ed. Biblioteca Nueva, 2012.

RIESTRA, D. El trabajo docente en la enseñanza de 
la lengua: los textos y el análisis entre los géneros y los tipos de discurso. en: CASTEL, V y Cubo de Severino, L, Los colores de la mirada lingüística. Mendoza Ed. de la Universidad Nacional de Cuyo, 2010, p. 1129-1138.

RIESTRA, D. Enseñar a razonar en lengua materna, las implicancias discursivas y textuales de la acción de lenguaje. Estudos Linguísticos / Linguistic Studies $n^{\circ}$ 3, (Lisboa: Colibri) - Revista do Centro de Linguística da Universidade Nova de Lisboa II. Faculdade de Ciencias Sociais e Humanas, UNL, Lisboa, Portugal, 2008, p. 411-425.

RIESTRA, D. Problemas actuales en Didáctica de la lengua y la literatura. Anales de Lingüística. Instituto de Lingüística. Facultad de Filosofía y Letras. Universidad Nacional de Cuyo, tomos XXXXXXI 2008-2009, (Sección Discursos y conferencias), 2011a, p. 37-51.

RIESTRA, D. La formación de los profesores de Lengua y Literatura y La enseñanza de los géneros textuales. Scripta, Belo Horizonte, v.15, n.28, $1^{\circ}$ sem., 2011 b, p.13-36.

RIESTRA, D. Descrição de um projeto (ou proposta) de formação continuada na Argentina.Formação continuada em ação: da base teórica ao domínio tecnológico. COSTA-
HÜBES, Terezinha da Conceição; MOLIN, Beatriz Helena Dal. (org). Cascavel: EDUNIOESTE. 2012, p. 45-61.

SCHNEUWLY, B. Genres et types de discours: considérations psychologiques et ontogénétiques, en Les interactions lecture-écriture. Actes du colloque Théodile -Crel (Lille, nov. 1993). Bern: Peter Lang, 1994.

SETTON, J. La literatura en: ALVARADO, M. Problemas de enseñanza de la lengua y la literatura. Bernal: Univ. de Quilmes, 2004.

VIVANTE, M. D. Dinámicos y curiosos. Contexto situacional. Didáctica de la literatura Bs.As.: Ed. Magisterio del Río de la Plata, 2006.

VOLOSHINOV, V. Marxismo y filosofía del lenguaje. Buenos Aires: Godot, Cap. II y III de la $1^{\text {a }}$ parte, 2009.

VYGOTSKI, L. Psicología pedagógica, Buenos Aires: Aique, 1926/2005.

VYGOTSKI, L. La imaginación y la creatividad del adolescente. Escritos sobre arte y educación creativa de Lev Vygotski. Madrid: Infancia \& Aprendizaje, 1931/2007.

\section{Sobre a autora:}

Dora Riestra: É Profesora Titular Ordinaria de Didáctica de la Lengua y la Literatura, Universidad Nacional de Río Negro, Argentina.

Artigo recebido em novembro de 2013

Artigo aprovado em fevereiro de 2014 